Delft University of Technology

\title{
A -109.1 dB/-98 dB THD/THD+N Chopper Class-D Amplifier with >83.7 dB PSRR Over the Entire Audio Band
}

Zhang, Huajun; Rozsa, Nuriel ; Berkhout, Marco; Fan, Qinwen

DOI

10.1109/ESSCIRC53450.2021.9567786

Publication date

2021

Document Version

Accepted author manuscript

Published in

ESSCIRC 2021 - IEEE 47th European Solid State Circuits Conference (ESSCIRC)

\section{Citation (APA)}

Zhang, H., Rozsa, N., Berkhout, M., \& Fan, Q. (2021). A -109.1 dB/-98 dB THD/THD+N Chopper Class-D Amplifier with >83.7 dB PSRR Over the Entire Audio Band. In ESSCIRC 2021 - IEEE 47th European Solid State Circuits Conference (ESSCIRC): Proceedings (pp. 395-398). [9567786] IEEE .

https://doi.org/10.1109/ESSCIRC53450.2021.9567786

Important note

To cite this publication, please use the final published version (if applicable).

Please check the document version above.

\footnotetext{
Copyright

Other than for strictly personal use, it is not permitted to download, forward or distribute the text or part of it, without the consent of the author(s) and/or copyright holder(s), unless the work is under an open content license such as Creative Commons.

Takedown policy

Please contact us and provide details if you believe this document breaches copyrights.

We will remove access to the work immediately and investigate your claim.
} 


\title{
A $-109.1 \mathrm{~dB} /-98 \mathrm{~dB}$ THD/THD+N Chopper Class- D Amplifier with $>83.7 \mathrm{~dB}$ PSRR Over the Entire Audio Band
}

\author{
Huajun Zhang ${ }^{1}$, Nuriel Rozsa ${ }^{1}$, Marco Berkhout ${ }^{2}$, Qinwen Fan ${ }^{1}$ \\ ${ }^{1}$ Department of Microelectronics, Delft University of Technology, Delft, The Netherlands \\ ${ }^{2}$ Goodix Technology, Nijmegen, The Netherlands \\ Email: h.zhang-13@tudelft.nl,n.n.m.rozsa@tudelft.nl, mberkhout@goodix.com, q.fan@tudelft.nl
}

\begin{abstract}
This paper reports a chopper Class-D audio amplifier that obtains high PSRR over the entire audio band. A chopping scheme is proposed to minimize intermodulation distortion between pulse-width modulation (PWM) and chopping in the audio band. A high-voltage chopper is developed to handle a 14.4 V PWM signal. Timing matching techniques are proposed to minimize chopping nonidealities which ensure good PSRR and THD. Fabricated in a $180 \mathrm{~nm}$ BCD process, the prototype obtains a PSRR $>109 \mathrm{~dB}$ at $217 \mathrm{~Hz}$ and $>83.7 \mathrm{~dB}$ over the entire audio band. It also achieves $-109.1 \mathrm{~dB} /-98 \mathrm{~dB}$ THD/THD $+\mathrm{N}$ and can deliver a maximum of $13 \mathrm{~W}$ to an $8-\Omega$ load.
\end{abstract}

Keywords-Class-D amplifier, chopping, PSRR, PWM, intermodulation

\section{INTRODUCTION}

Class-D amplifiers (CDAs) achieve high power efficiency because their output is switched between the supply and ground. To improve supply rejection and linearity, they often employ a closed-loop configuration with resistive feedback. However, the mismatch between feedback resistors causes common-mode-to-differential leakage and limits the overall PSRR. To achieve $>100 \mathrm{~dB}$ PSRR, the mismatch between feedback resistors should be in the order of $10 \mathrm{ppm}$, which would require excessive die area or expensive trimming for high yield. The PSRR can also be improved by filtering the supply off-chip at the expense of increased system size. In [1, $2]$, an output common-mode regulation loop is employed to improve PSRR, but the PSRR drops significantly towards the edge of the audio band $(20 \mathrm{kHz})$.

Alternatively, PSRR can be improved by removing feedback resistor mismatch using chopping. However, incorporating chopping into a pulse-width modulation (PWM) high-voltage (HV) CDA [2-5] is challenging. First, intermodulation between chopping and PWM in the audio band must be minimized. Second, an HV chopper capable of processing the HV fast-switching PWM signal at the CDA output is required. Third, chopping nonidealities such as duty cycle errors and glitches should be minimized to ensure good PSRR and THD.

This paper presents a $14.4 \mathrm{~V}$ chopper Class-D amplifier that addresses these challenges. Section II introduces the architecture of the CDA and explains the chopping scheme proposed to minimize the intermodulation between chopping and PWM in the audio band. Section III describes the implementation of the HV chopper and the timing alignment techniques to reduce chopping artifacts. Measurement results are reported in Section IV, followed by conclusions drawn in Section V.

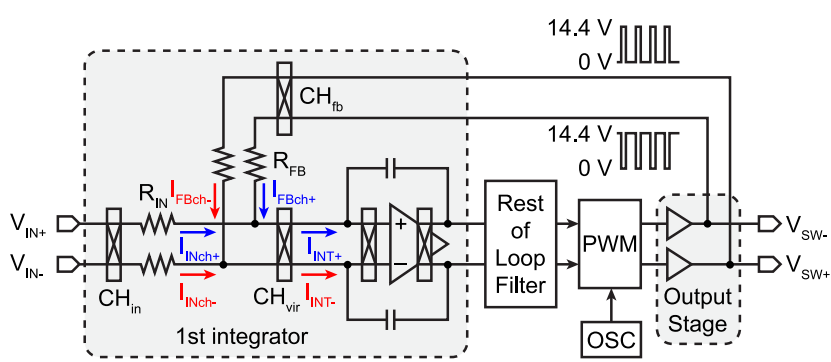

Fig. 1. Architecture of the proposed chopper CDA.
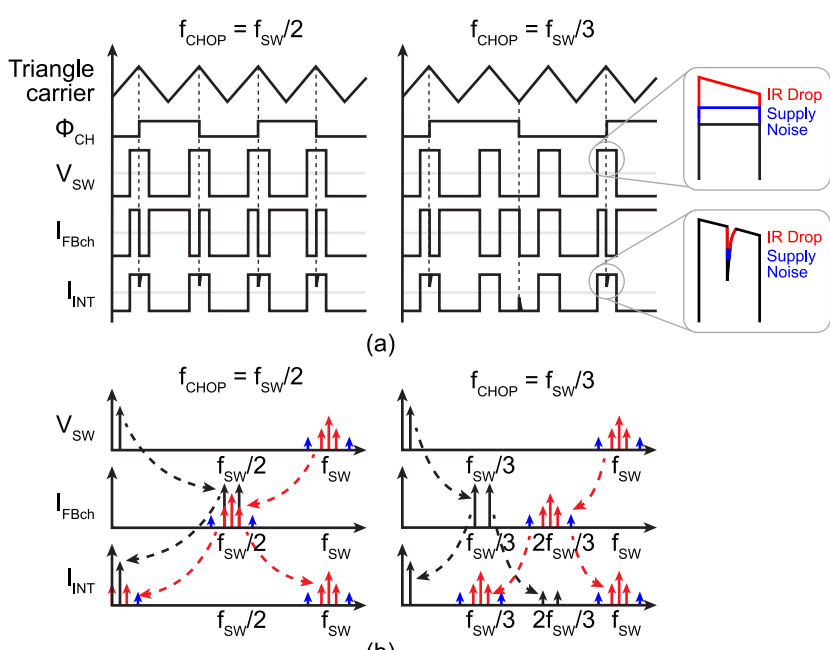

(b)

Fig. 2. (a) Time and (b) frequency domain behavior of chopping a PWM signal.

\section{ARCHITECTURE}

\section{A. Overview}

Fig. 1 shows the architecture of the chopper CDA. It consists of a $3^{\text {rd }}$ order loop filter and a PWM modulator in the $1.8-\mathrm{V}$ domain, a fully differential $\mathrm{HV}$ output stage, and a resistive feedback network defining a closed-loop gain of 8 . The input and feedback signals are modulated by the choppers $\mathrm{CH}_{\text {in }}$ and $\mathrm{CH}_{\mathrm{fb}}$, respectively, and then demodulated by $\mathrm{CH}_{\text {vir. }}$ The OTA of the $1^{\text {st }}$ integrator is also chopped, which removes its $1 / f$ noise and increases its CMRR, which rejects the HV supply-induced common-mode (CM) variations at its virtual ground.

\section{B. Choice of Chopping Frequency}

To minimize intermodulation between PWM and chopping in the audio band, the chopping scheme must be carefully chosen. In this work, the chopping clock and the 


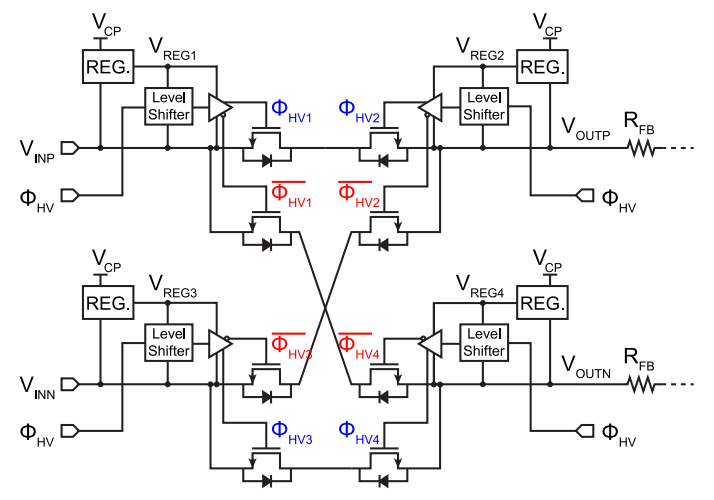

Fig. 3. Schematic of the HV chopper.

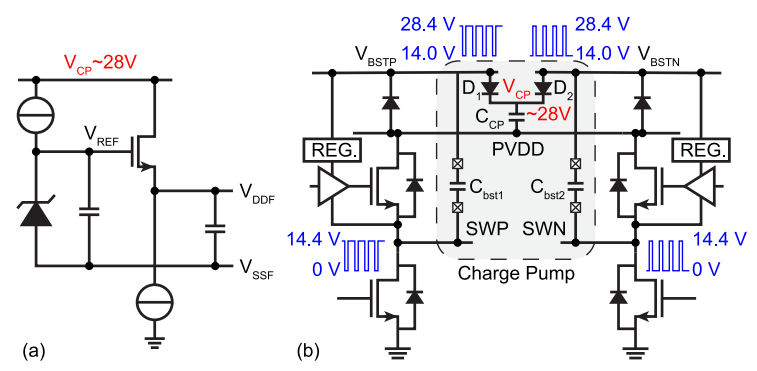

Fig. 4. (a) Regulator used in HV chopper, and (b) CDA output stage and the charge pump that supplies the regulators in the HV chopper.

triangle wave carrier for PWM generation are synchronized such that the chopping transitions always occur at the center of a PWM pulse, as shown in Fig. 2(a). This avoids having very narrow pulses in $\mathrm{I}_{\mathrm{FBch}}$, which can be easily filtered by parasitic capacitances, leading to distortion. Moreover, chopping produces glitches at the switching transitions, which introduce two signal-dependent error sources and can degrade both PSRR and THD. The first error source is the supply noise (shown in blue). The second error source is the IR drop across the output stage power transistors (shown in red). When the class D amplifier drives a speaker, which can be electrically modeled as an inductor in series with a resistor, a signaldependent ripple current is produced, which in turn creates a ripple voltage across the finite on-resistance of the output power transistors. The IR drop, therefore, contains PWM signal content that exhibits sideband around multiples of the PWM switching frequency $f_{P W M}$ as shown in Fig. $2 b$ [6]. To minimize degradation in both PSRR and THD due to intermodulation, the chopping frequency $\mathrm{f}_{\mathrm{CH}}$ is chosen to be an odd subharmonic rather than an even subharmonic of $f_{S w}$. The two scenarios are compared in Fig. 2 in both the time and frequency domains. When the ratio $\mathrm{f}_{\mathrm{SW}} / \mathrm{f}_{\mathrm{CH}}$ is even (e.g., equal to 2), the chopping glitch errors appear in the baseband, degrading both PSRR and THD. In contrast, when the ratio $\mathrm{f}_{\mathrm{SW}} / \mathrm{f}_{\mathrm{CH}}$ is odd (e.g., equal to 3 ), the glitch errors are modulated to the chopping frequency. Choosing $\mathrm{f}_{\mathrm{CH}}$ to be higher than the audio band edge $(20 \mathrm{kHz})$ with a sufficient margin guarantees that the demodulated PWM sideband around $\mathrm{f}_{\mathrm{CHOP}}$ is kept out of the audio band. In this work, an $\mathrm{f}_{\mathrm{CH}}$ of $100 \mathrm{kHz}\left(=\mathrm{f}_{\mathrm{Sw}} / 21\right)$ is chosen. It should be pointed out that the IR drop also contains components around even multiples of $\mathrm{f}_{\mathrm{SW}}$, which will be modulated to the baseband. To minimize this intermodulation, the total energy of the glitches should be reduced, which can be done by choosing a low $\mathrm{f}_{\mathrm{CH}}$ and using circuit techniques to reduce the size of the individual glitch, as described in Section III.
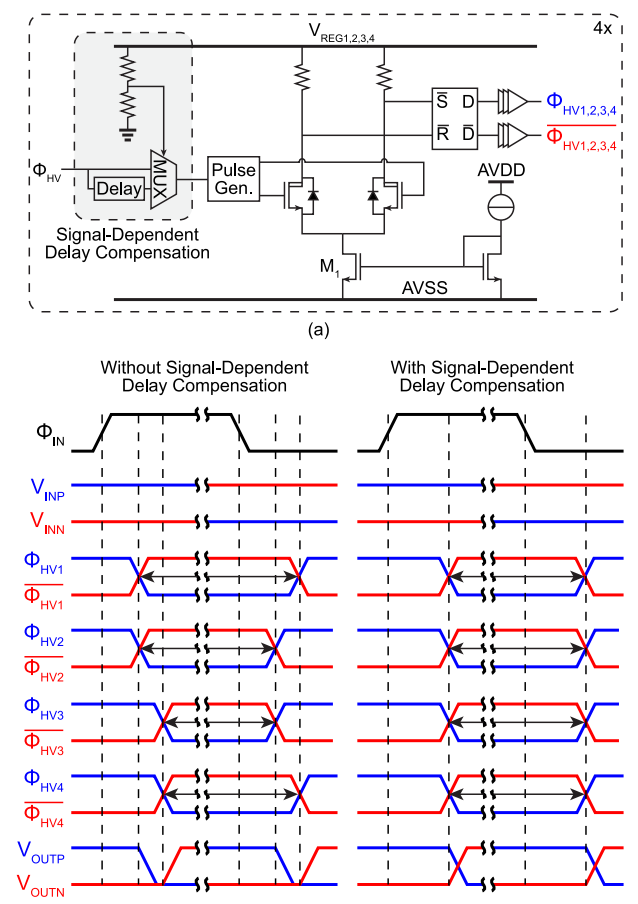

(b)

Fig. 5. (a) Level shifter [7] with added signal-dependent delay compensation circuit, and (b) input, output, and switch control signals in the HV chopper with and without compensation for the signal-dependent delay.

\section{CIRCUIT IMPLEMENTATION}

\section{A. High-Voltage Chopper}

To handle the 14.4 V PWM signal, a bootstrapped HV chopper $\left(\mathrm{CH}_{\mathrm{fb}}\right)$ is proposed, as shown in Fig. 3. Back-to-back LDMOS transistors are used to block the conduction path through the body diodes when the switches are turned off. The chopper clocks are transferred from the LV digital domain $(1.8 \mathrm{~V})$ to the HV domain by level shifters [7], each powered by a local regulator. All regulators are sourced by a charge pump that provides a $\sim 28 \mathrm{~V}$ supply.

Each open-loop regulator, shown in Fig. 4a, employs a Zener reference and a source follower output stage. The charge pump, shown in Fig. 4b, consists of 2 Schottky diodes $\left(\mathrm{D}_{1}\right.$ and $\left.\mathrm{D}_{2}\right)$, an on-chip capacitor $\mathrm{C}_{\mathrm{CP}}$, and reuses two off-chip bootstrap capacitors $\mathrm{C}_{\mathrm{bst} 1}$ and $\mathrm{C}_{\mathrm{bst} 2}$ that are already required in the floating gate driver circuits of the power transistors [5]. $\mathrm{C}_{\mathrm{CP}}$ is charged by $\mathrm{C}_{\mathrm{bst} 1}$ and $\mathrm{C}_{\mathrm{bst} 2}$ in an alternating fashion in each PWM cycle, holding $\mathrm{V}_{\mathrm{CP}}$ up to $\sim 28 \mathrm{~V}$. The level shifter is shown in Fig. 5a [7]. Depending on the PWM signal and the chopping phase, $\mathrm{V}_{\mathrm{REG1} 1,2,3,4}$ are either $\sim 5 \mathrm{~V}$ or $\sim 19.4 \mathrm{~V}$. This introduces a signal-dependent delay in the level shifter since, in the former case, the bias current source $\mathrm{M}_{1}$ operates in the triode region for part of the transition. Therefore, the chopping signals experience a longer delay when switching from $0 \mathrm{~V}$ to 14.4 $\mathrm{V}$ and a shorter delay vice versa. As a result, two distinct switching transitions occur, as illustrated in Fig. 5b, which causes a duty cycle error that degrades PSRR. To mitigate this issue, a delay compensation block is employed in the HV chopper drivers. A simple 10:1 resistor divider detects the voltage level of $V_{\mathrm{REG}}$ and either enables a delay if $\mathrm{V}_{\mathrm{REG}} \approx 19.4$ $\mathrm{V}$ or disables the delay if $\mathrm{V}_{\mathrm{REG}} \approx 5 \mathrm{~V}$ (Fig. 5a) before the next transition. As a result, all HV chopper switching transitions are aligned. 

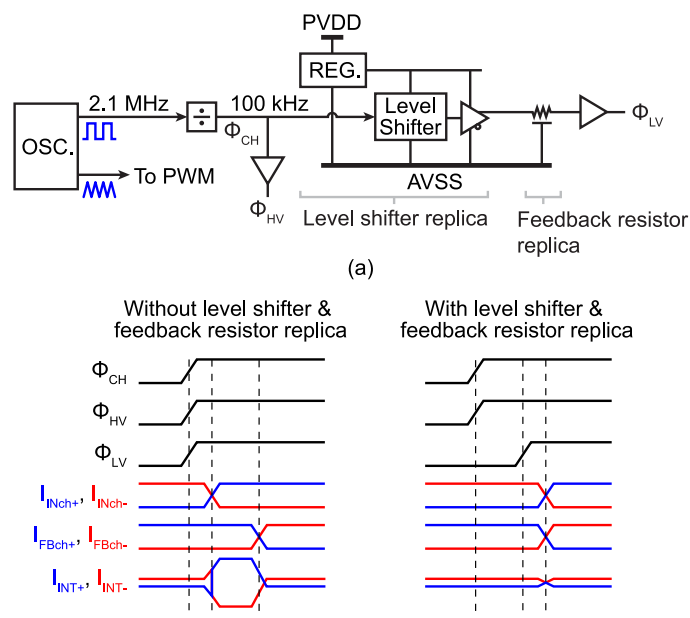

With level shifter \& feedback resistor replica
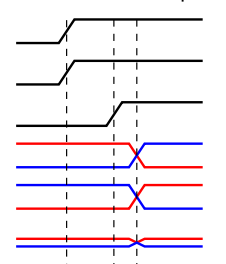

(b)

Fig. 6. (a) Replica delay circuit to align chopping transitions of HV and LV choppers, and (b) HV and LV chopper clock signals and resulting input, feedback, and integrator current with and without the replica delay circuit.

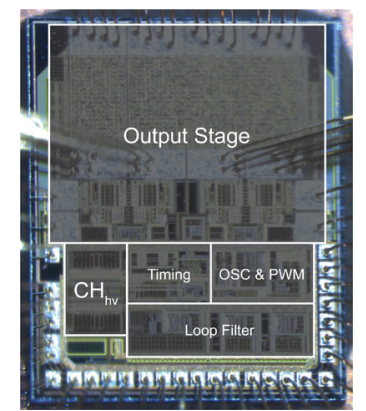

Fig. 7. Die photo.

\section{B. HV and LV Chopper Timing Matching}

Apart from $\mathrm{CH}_{\mathrm{fb}}$, all the other choppers are implemented in the $1.8 \mathrm{~V}$ domain. Timing skew between the HV and LV choppers due to different driving circuitries results in large chopping glitches that can degrade THD and PSRR. Moreover, the parasitic capacitance in the feedback resistors adds a delay between $\mathrm{CH}_{\mathrm{fb}}$ and $\mathrm{CH}_{\mathrm{vir}}$, which again causes large chopping glitches. Replicas for the level shifter and feedback resistor, shown in Fig. 6a, are added in the LV chopper driving path to mitigate these issues across PVT variations. Fig. $6 \mathrm{~b}$ illustrates the chopped input current $\mathrm{I}_{\mathrm{INch}+/ \text {-, }}$ chopped feedback current $\mathrm{I}_{\mathrm{FBch}+/-}$, and the current going into the $1^{\text {st }}$ integrator $\mathrm{I}_{\mathrm{INT}+/}$ (indicated in Fig. 1) with and without the delay replicas. As shown, the replicas can significantly remove the timing skew and therefore minimize the chopping glitches in the current flowing into the $1^{\text {st }}$ integrator.

\section{LV Circuitry}

To ensure linearity with a full-scale input signal, the input choppers are bootstrapped to maintain a constant on- $\mathrm{V}_{\mathrm{GS}}$ for the switches [8]. A PWM modulator driven by an on-chip oscillator runs at $2.1 \mathrm{MHz}$, which allows a relatively high loop gain for high linearity. A $3^{\text {rd }}$ order loop filter $[5,7]$ is employed, which provides $>80 \mathrm{~dB}$ loop gain within the audio band to suppress the distortion of the output power stage. The input stage of the $1^{\text {st }}$ integrator's OTA is sized such that its $1 / f$ noise corner frequency is below $\mathrm{f}_{\mathrm{CHOP}}$.

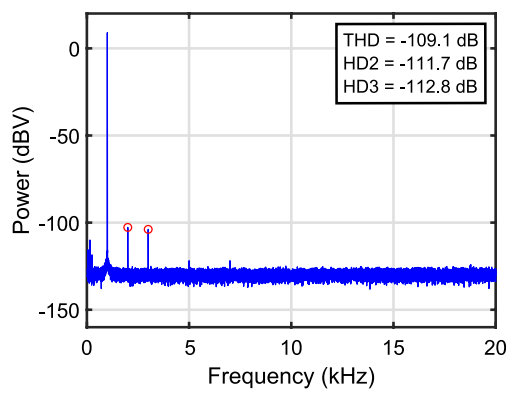

Fig. 8. Output spectrum for $1 \mathrm{kHz}$ input at $1 \mathrm{~W}$ output power.

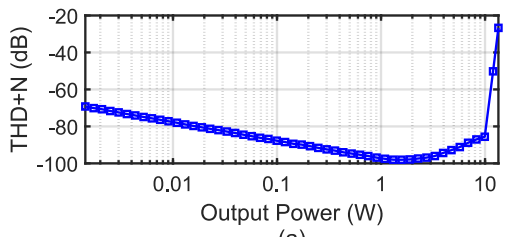

(a)

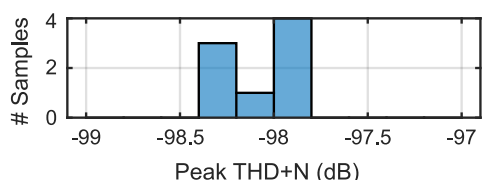

(b)

Fig. 9. (a) THD+N across output power, and (b) peak THD $+\mathrm{N}$ for 8 samples.

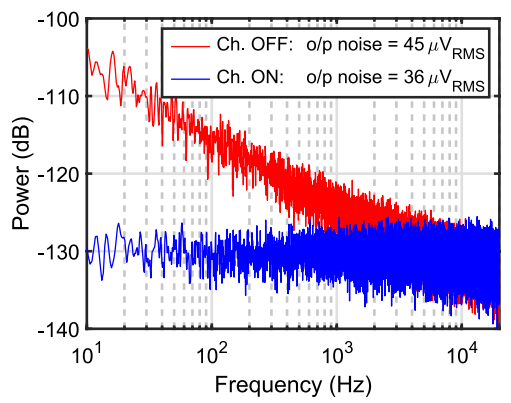

Fig. 10. Output spectra with and without chopping with a zero input.

\section{Measurement Results}

The design is prototyped in a $180 \mathrm{~nm}$ BCD process and occupies $5 \mathrm{~mm}^{2}$ (Fig. 7). The HV chopper, its driving circuit (the level shifters and regulators), and the timing replica circuits occupy $7 \%$ of the total chip area. For all measurements, the CDA is loaded with $(44 \mu \mathrm{H}+8 \Omega)$, which mimics a real speaker, and the output is measured using an Audio Precision APx555 Analyzer.

The measured output spectrum for a $1 \mathrm{kHz}$ sine wave input is shown in Fig. 8. The CDA achieves a THD of $-109.1 \mathrm{~dB}$ when delivering $1 \mathrm{~W}$ into the load. The THD+N performance across output power is plotted in Fig. 9. The CDA achieves a peak THD $+\mathrm{N}$ of $-98 \mathrm{~dB}(0.0013 \%)$. Fig. 10 compares the output spectra with a zero input when chopping is turned $\mathrm{ON}$ and OFF. With chopping ON, flicker noise is clearly suppressed, and the integrated output noise (A-weighted) improves from $45 \mu \mathrm{V}_{\text {RMS }}$ to $36 \mu \mathrm{V}_{\text {RMS }}$. Fig. 11 plots the PSRR across the entire audio band for 8 samples measured by superimposing a $2 \mathrm{~V}_{\mathrm{PP}}$ sinewave onto the $14.4 \mathrm{~V}$ supply. When chopping is turned OFF, the PSRR spreads widely 
TABLE I. PERformance SumMARY AND COMPARISON.

\begin{tabular}{|c|c|c|c|c|c|}
\hline & This Work & D. Schinkel [2] & {$[3]$} & E. Cope [4] & S. Karmakar [5] \\
\hline Area (mm $\left.{ }^{2}\right)$ & 5 & - & - & 4.3 & 4.8 \\
\hline Supply (V) & 14.4 & 25 & 14.4 & $8 \sim 20$ & 14.4 \\
\hline$f_{S W}(\mathrm{kHz})$ & 2100 & 500 & 2100 & 400 & 2000 \\
\hline Quiescent Current (mA) & 21 & - & 180 & 20.5 & 17 \\
\hline Idle Power (mW) & 302 & - & - & - & 245 \\
\hline Output Power (W) & 13 & 80 & 75 & 20 & 28 \\
\hline Efficiency & $92 \%$ & $>90 \%$ & $86 \%$ & $90 \%$ & $91 \%$ \\
\hline Peak THD+N @ 1kHz & $0.0013 \%$ & $0.004 \%$ & $0.02 \%$ & $0.0013 \%$ & $0.0008 \%$ \\
\hline SNR (A-weighted) & $108 \mathrm{~dB}$ & - & - & $116 \mathrm{~dB}$ & - \\
\hline DR (A-weighted) & $110.3 \mathrm{~dB}$ & $115 \mathrm{~dB}$ & - & $115.5 \mathrm{~dB}$ & $109 \mathrm{~dB}$ \\
\hline PSRR & $109 \mathrm{~dB} \sim 83 \mathrm{~dB}$ & $88 \mathrm{~dB} \sim 60 \mathrm{~dB}$ & $75 \mathrm{~dB} \sim 57 \mathrm{~dB}$ & $80 \mathrm{~dB} \sim 50 \mathrm{~dB}$ & $70 \mathrm{~dB} \sim 62 \mathrm{~dB}$ \\
$($ Frequency Range) & $(20 \mathrm{~Hz} 20 \mathrm{kHz})$ & $(100 \mathrm{~Hz} \sim 20 \mathrm{kHz})$ & $(20 \mathrm{~Hz} \sim 20 \mathrm{kHz})$ & $(20 \mathrm{~Hz} 20 \mathrm{kHz})$ & $(20 \mathrm{~Hz} \sim 20 \mathrm{kHz})$ \\
\hline \# Samples & 8 & - & - & & - \\
\hline
\end{tabular}

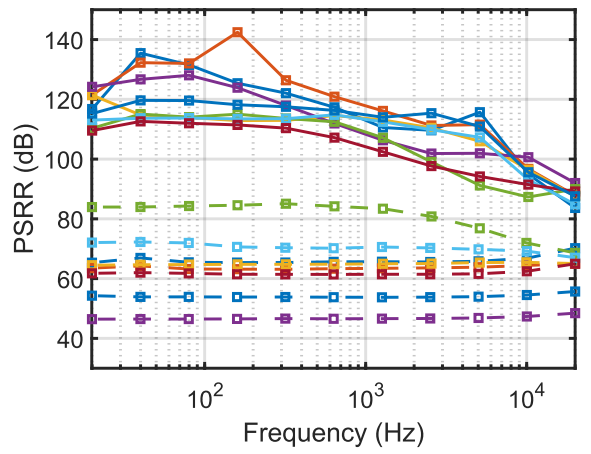

(a)

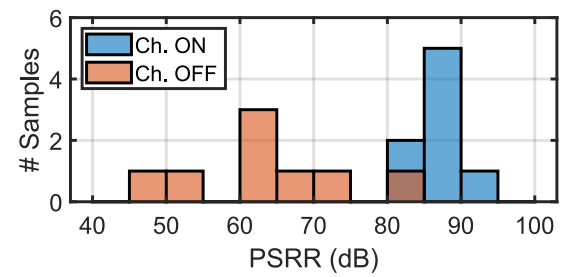

(b)

Fig. 11. (a) PSRR across the audio band with chopping ON (solid) and OFF (dashed), and (b) Comparison of PSRR at $20 \mathrm{kHz}$ between chopping ON and OFF.

between $46 \mathrm{~dB}$ and $84 \mathrm{~dB}$ due to random resistor mismatch. With chopping enabled, the PSRR is improved to $>109 \mathrm{~dB}$ at low frequencies for all samples and remains $>83 \mathrm{~dB}$ across the entire audio band. Fig. 12 shows the efficiency across output power. The CDA achieves a peak efficiency of $91.8 \%$. It draws a quiescent current of $22 \mathrm{~mA}$ from the $14.4 \mathrm{~V}$ supply.

Table I summarizes the performance of this CDA and compares it with other state-of-the-art HV $(>10 \mathrm{~V})$ CDAs. This work achieves over $20 \mathrm{~dB}$ higher PSRR both at low frequencies and $20 \mathrm{kHz}$ while attaining competitive THD+N, SNR, and power efficiency.

\section{CONCLUSION}

This paper proposes the use of chopping to improve the PSRR of CDAs. The chopping frequency is carefully chosen to be an odd subharmonic of the PWM switching frequency to minimize intermodulation between chopping and PWM in the audio band. An HV chopper capable of processing the $14.4 \mathrm{~V}$ CDA output is presented. Timing alignment techniques are proposed to reduce chopping duty cycle errors and glitches to ensure good THD and PSRR. Measurement results across multiple samples show a $>20 \mathrm{~dB}$ improvement in PSRR over

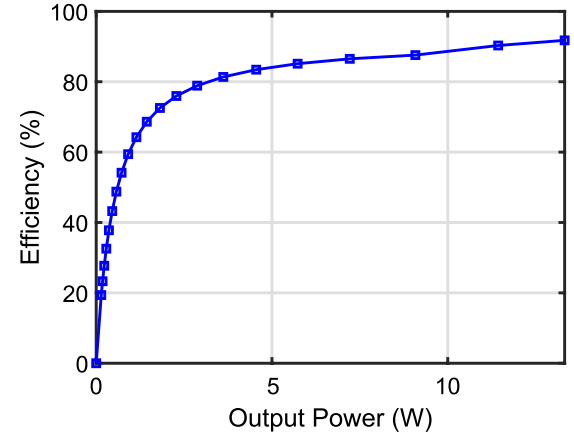

Fig. 12. Power efficiency across output power.

the entire audio band while achieving competitive THD $+\mathrm{N}$ performance compared to other state-of-the-art designs.

\section{REFERENCES}

[1] W. Wang and Y. Lin, "A 118 dB PSRR, $0.00067 \%$ (-103.5 dB) THD+N and 3.1 W Fully Differential Class-D Audio Amplifier With PWM Common Mode Control," IEEE J. Solid-State Circuits, vol. 51, no. 12, pp. 2808-2818, Dec. 2016.

[2] D. Schinkel et al., "A Multiphase Class-D Automotive Audio Amplifier With Integrated Low-Latency ADCs for Digitized Feedback After the Output Filter," IEEE J. Solid-State Circuits, vol. 52, no. 12, pp. 31813193, Dec. 2017.

[3] Texas Instruments, "TAS6424-Q1 75-W, 2-MHz Digital Input 4Channel Automotive Class-D Audio Amplifier With Load-Dump Protection and I2C Diagnostics," Accessed on: July 15, 2020. [Online]. Available: https://www.ti.com/lit/gpn/tas6424-q1

[4] E. Cope et al., "A $2 \times 20 \mathrm{~W} 0.0013 \%$ THD+N Class-D audio amplifier with consistent performance up to maximum power level," in IEEE Int. Solid-State Circuits Conf. (ISSCC) Dig. Tech. Papers, Feb. 2018, pp. 56-58.

[5] S. Karmakar et al., "A 28-W, -102.2-dB THD+N Class-D Amplifier Using a Hybrid $\triangle \Sigma \mathrm{M}-\mathrm{PWM}$ Scheme," IEEE J. Solid-State Circuits, vol. 55, no. 12, pp. 3146-3156, Dec. 2020.

[6] R. A. Guinee and C. Lyden, "A single Fourier series technique for the simulation and analysis of asynchronous pulse width modulation in motor drive systems," in ISCAS '98. Proceedings of the 1998 IEEE International Symposium on Circuits and Systems (Cat. No.98CH36187), 1998, vol. 6, pp. 653-656 vol.6.

[7] H. Zhang et al., "A High-Linearity and Low-EMI Multilevel Class-D Amplifier," IEEE J. Solid-State Circuits, vol. 56, no. 4, pp. 1176-1185, Apr. 2021.

[8] C. Liu, S. Chang, G. Huang, and Y. Lin, "A 10-bit 50-MS/s SAR ADC With a Monotonic Capacitor Switching Procedure," IEEE J. Solid-State Circuits, vol. 45, no. 4, pp. 731-740, Apr. 2010. 